\title{
ACUTE DISSEMINATED LANGERHANS CELL DISEASE - A RARE CASE REPORT
}

Sunitha. M, R. Rajesh

1. Professor, Department of Oral Medicine \& Radiology, Noorul Islam Dental College, Thiruvananthapuram, Kerala

2. Professor, Department of Pedodontics, Indira Gandhi Dental College, Kothamangalam, Ernakulam Dist, Kerala.

\section{CORRESPONDING AUTHOR}

Dr. Sunitha. M.

T.C. 25/3361, Chirakulam Road

Thiruvananthapuram, Kerala,

E-mail: rajeshkttytvm2005@yahoo.co.in

Ph: 00919495200704.

ABSTRACT: Langerhans cell disease previously known as Histiocytosis $X$ is a non lipidreticuloendotheliosis that presents with single or multiple lesions distributed in the soft tissues, lymph nodes, various organs and bones, especially in areas of marked reticulo endothelial activity. Histiocytosis X encompasses three conditions which include a localized form (Eosinophilic granuloma), a chronic disseminated form (Hand Schuller-Christian disease) and an acute or subacute disseminated form (Letterer-Siwe disease). Eosinophilic granuloma is characterized by solitary or multiple skeletal lesions with no extra skeletal involvement whereas Hand Schuller- Christian disease manifests with widespread skeletal and extra skeletal lesions with a chronic clinical course. Letterer-Siwe disease presents with widespread lesions of both skeletal and extra skeletal tissues with a more acute severe clinical course and a high mortality rate. Most often these patients report initially to the dentist because of the significant oral involvement including gingival swelling and mobility of teeth. Such a rare case of acute disseminated Langerhans cell disease that presented to the dental clinic is discussed in this article.

KEY WORDS: Letterer-Siwe,Histiocytosis X, Langerhans cell disease.

INTRODUCTION: Acute disseminated Langerhans cell disease belongs to a group of diseases once known as Histiocytosis X. The term Histiocytosis X was suggested by Lichtenstein in 1953 which included a spectrum of disorders characterized by proliferation of histiocyte- like cells ${ }^{1}$. These disorders include Letterer-Siwe disease, Hand Schuller-Christian disease and Eosinophilic granuloma. The term Langerhans cell disease is preferred to the older term Histiocytosis $\mathrm{X}$ as it emphasizes the histogenesis of the condition by specifying the type of lesional cells and removes the connotation of the unknown ' $X$ ' because its cellular basis has now been clarified. The Working Group of the Histiocyte society has divided histiocytic disorders into 3 different groups 1) dendritic cell histiocytosis 2) erythrophagocytic macrophage disorders and 3) malignant histiocytosis. Langerhans cell disease belongs to group 1 and encompasses a number of diseases ${ }^{2}$. The clinical spectrum includes on one end, an acute fulminant disseminated disease called Letterer-Siwe disease and on the other end, solitary or few indolent chronic lesions of bone and other organs called Eosinophilic granuloma. The intermediate clinical form called Hand Schuller-Christian disease is characterized by multifocal chronic involvement and classically presents as a triad of diabetes insipidus, proptosis and lytic 


\section{CASE REPORT}

bone lesions. The acute to subacute form of disseminated Langerhans cell disease is the most severe form and generally affects infants, the age of onset being less than 3 years. The disease may run a rapid course and is often fatal.

CASE REPORT: A 3 year old male patient reported to the dental clinic with complaint of growth over gums and displaced teeth and irritation of the ears since 3 months. The growth started as a localized lesion and rapidly increased to the present size. On General examination, the patient had fever and exhibited irritability. On extra oral examination, face was symmetrical and the left submandibular lymphnodes were enlarged. Crustation of upper and lower lip was seen. On intra oral examination, all the deciduous teeth were present. There was a diffuse growth of the upper gingiva and palate from anterior to the posterior region (Fig.1). The growth was erythematous and there was severe displacement of teeth with 53 and 63 displaced palatally and the teeth showed mobility. The growth was soft, tender and there was bleeding on palpation. Growth was also seen involving the entire lower gingiva and there was displacement and mobility of the lower teeth as well. Lab investigations revealed anemia, bleeding and clotting time and other blood parameters were within normal limits. Mantoux test was negative. Peripheral smear showed that the RBC's were hypochromic and microcytic and no evident immature cells. Panoramic radiograph showed large radiolucent areas in maxilla and mandible with teeth showing a 'floating in air appearance'. Lateral Skull view showed large radiolucent lytic lesions in the skull (Fig.2). Chest $X$ ray and $X$ ray of upper and lower limbs revealed no evident radiolucent lytic lesions. There was no evident hepatosplenomegaly. Biopsy was done and histopathologically the lesion showed proliferation of histiocytes with large pale cytoplasm containing small dark granules (Fig 3). Correlating the history, clinical, radiologic examination and histopathologic findings a final diagnosis of acute disseminated Langerhans cell disease was made. The patient was referred to a higher centre where chemotherapy was planned. But the patient refused to undergo treatment and opted for 'Yunani' treatment at his home town. The patient didn't turn up for recall and 5 years after diagnosis the patient was reported to be well (after a telephonic conversation) and was attending school.

DISCUSSION: Histiocytosis $\mathrm{X}$ has been defined as a non neoplastic, proliferative disorder of histiocytes of unknown etiology, which is pathogenetically similar to an inflammatory reaction with multiple clinical manifestations mirroring the widespread distribution of the histiocytic system of the body3. In 1924, Letterer reported the case of an infant who presented with hepatosplenomegaly, anemia and a purpuric eruption of the skin. Autopsy showed a proliferation of reticulo endothelial cells that replaced the normal structure of several organs and called the condition 'Aleukemicreticulosis'. In 1933 Siwe grouped several reported cases similar to that described by Letterer and one of his own into a well defined clinical-pathologic syndrome under the name of 'Reticuloendotheliosis'. Abt and Denenholz in 1936, clearly delineated and differentiated this disease from the other so- called reticuloendothelioses, employing for the first time the term, 'Letterer-Siwe disease'4. A para colon bacillus from the Arizona group was isolated by Fisher in 1953 from a patient presenting with clinical signs and symptoms of Letterer- Siwe disease ${ }^{5}$. Antibiotic therapy was followed by a successful response. Farber first proposed the theory that Letterer-Siwe disease, Hand Schuller Christian disease and Eosinophilic granuloma of bone are all manifestations of a single disease ${ }^{6}$.

It has been demonstrated that the histiocytes present in the lesion are Langerhans cells which are dendritic mononuclear cells normally found in the epidermis, mucosa, lymph 


\section{CASE REPORT}

nodes and bone marrow which process and present antigens to T lymphocytes. For this reason, the term 'Histiocytosis $\mathrm{X}$ ' has now been largely replaced by the terminology 'Langerhans cell disease (LCD). Failure to detect aneuploidy or consistent karyotypic abnormalities supports the idea that LCD is not a neoplastic process or malignancy. However, the proliferation of Langerhans cells may be a physiological rather than a pathologic response. Aberrant or uncontrolled cytokine production is known to play a role in reactive histiocyte disorders.

In acute disseminated LCD, young children are most frequently affected, even though adults may also be affected ${ }^{7}$. The initial symptom generally is a skin rash scattered over the trunk and scalp and in less severe forms over the extremities. Otitis media and purulent discharge from eyes are also early manifestations. The patient has high fever, malaise and irritability. Splenomegaly and/or hepatomegaly as well as lymphadenopathy may be evident early in the course of the disease or shortly after the initial signs or symptoms. The lungs and gastrointestinal tract are affected as well. There is diffuse skeletal involvement later in the course of the disease with large radiolucent lesions.

Oral manifestations may be among the earlier signs of disseminated or localized LCD. Patients have swelling over the gingiva, halitosis, mobile teeth, and bad taste in mouth. Children may have premature exfoliation of deciduous teeth followed by eruption of permanent teeth which also become mobile. The appearance of advanced periodontal disease in young children may be suggestive of Langerhans cell disease. The failure of a socket to heal after extraction of a mobile tooth is another significant sign. Radiographic examination reveals a non specific radiolucent appearance. There is alveolar bone resorption in the molar and premolar region. The anterior regions of the jaw are usually involved only in the later course of the disease. The margins of individual lesions may be well defined or indistinct. Perforation of the cortical plate is a common finding particularly in larger lesions. If the destruction is severe enough the teeth appear to have a 'floating in air'appearance. The skull lesions characteristically have a punched out appearance. Radiological examination of chest which has a pulmonary involvement usually reveals a disseminated nodular infiltration, often poorly defined and varying in size which are concomitant with pulmonary fibrosis and obstructive emphysema.

Lab studies reveal hematologic abnormalities like anemia and thrombocytopenia. Histologically, histiocytes are the predominant cells, characterized by large pale cytoplasm at times containing small dark granules. Eosinophils are present. Necrosis is especially common early in the disease. Plasma cells, lymphocytes and at times polymorphonuclear cells are also present. Ultra structurally, Langerhans cells can be demonstrated which contain rod shaped cytoplasmic structures called Birbeck granules. Birbeck granules are intracellular in location having a tennis racket appearance and identified by electron microscopy. Immunohistochemical studies are also helpful in confirming the presence of Langerhans cells. These cells stain positively for S-100 protein and they also show binding for peanut agglutinin(PNA) ${ }^{8}$. The presence of CD1a antigen on the cell surface and HLA-DR positivity confirms the Langerhans cell origin of this disease.

Acute leukemia can be differentiated from acute disseminated LCD by blood examination. Peripheral smear shows immature leukocytes in leukemia. Ewing's Sarcoma or malignant reticuloendotheliosis should be considered in the differential diagnosis. Diagnosis is established by histologic examination. Premature loss of teeth with bleeding gingiva could indicate Papillon- Lefevre syndrome, lymphosarcoma and several other conditions. Complete clinical examination, family history, blood examination and biopsy should lead to a proper diagnosis. 
Prognosis is usually poor for the acute disseminated form. Although the disease is not uniformly fatal and may run a protracted course, it has a high mortality rate from the organ involvement and secondary complications like hemorrhage and infection which may develop as a result of therapy. Radiation, alkylating agents, antimetabolites, steroids, vasopressin and antibiotics have been used with success in disseminated forms of the disease. Vinblastine sulfate, a cytotoxin produces good results as a therapeutic agent ${ }^{9}$. Lichtenstein, however has warned against the use of cytotoxins and radioactive isotopes in the acute disseminated disease for fear of further injury to an already damaged bone marrow. Antibiotics are useful in combating the secondary infection that may occur. Steroids, particularly prednisone has been valuable in helping suppress the disease symptoms during severe phases. Radiation therapy usually in the dosage range of 800-1500 rads has been used successfully in the treatment of localized mucocutaneous and skeletal lesions. Despite the low doses of radiation required for treatment, adverse effects like growth retardation, injury to developing teeth and the possibility of post irradiation neoplasia are common especially in young children. Thus the risk versus benefits of radiotherapy must be carefully weighed before starting the treatment procedure.

Yunani is a form of traditional medicine widely practiced in South Asia. It is based on the concept of the four humours: Phlegm, Blood, Yellow bile and Black bile. Yunani medicine is very close to Ayurveda. Both are based on the theory of the presence of elements (In Yunani they are fire, water, earth and air) in the human body. These elements are present in different fluids and their balance leads to health and their imbalance leads to illness. However, in recent days, awareness has been created regarding the safety and adverse effects of herbal drugs including Yunani drugs.

CONCLUSION: The role of the dentist in the diagnosis of Langerhans cell disease is emphasized as most cases present with oral findings and report initially to a dentist. A brief history of the disease and the evolution of the concept of grouping the three conditions under the term 'Histiocytosis $\mathrm{X}$ ' and the prevalent use of the new terminology 'Langerhans cell disease' has been reviewed. The role of alternative systems of medicine like Yunani in the management of proliferative disorders like LCD needs to be explored further which can open new vistas in the management and prognosis of the disease.

\section{REFERENCES:}

1. Lichtenstein L. Histiocytosis X. Integration of eosinophilic granuloma of bone, LettererSiwe disease and Schuller- Christian disease as related manifestations of a single Nosologic entity. Arch. Pathol. Lab. Med 1953; 56: 84-102.

2. The Working Group of the Histiocyte Society. Histiocytosis syndrome in children. Lancet.1987; 1:208-209.

3. Enriquez P et al. Histiocytosis X: a clinical study. Mayo Clin Proc. 1967; 42:88.

4. Abt A.F, Denenholtz E.J. Letterer-Siwe's disease: Splenomegaly associated with widespread hyperplasia of nonlipoid storing macrophages: Discussion of the so called Reticuloendothelioses. Am.J.Dis.Child. 1936; 51:499-582.

5. Fisher R.H. Multiple lesions of bone in Letterer- Siwe Disease: Report of case with culture of paracolon bacillus from bone lesions and blood. Followed by response to therapy. J. Bone \& Joint Surg. 1950; 35A: 443-464. 
6. Farber S.J. The nature of Solitary or Eosinophilic Granuloma of bone. Am.J.Path.1941; 17: 625-629.

7. Lichtenstein L. Histiocytosis X. J. Bone Joint Surg. 1964; 46A: 76.

8. Sane S.Y, Nainaney K.H. Histiocytosis X: The role of histology and S-100 protein in grading the severity of disease. J. Postgrad Med.1990; 36:16-19.

9. Beier F.R, Thatcher L.G, Lahey M.E. Treatment of reticuloendotheliosis with vinblastine sulfate. J. Pediat. 1963; 63:1087.

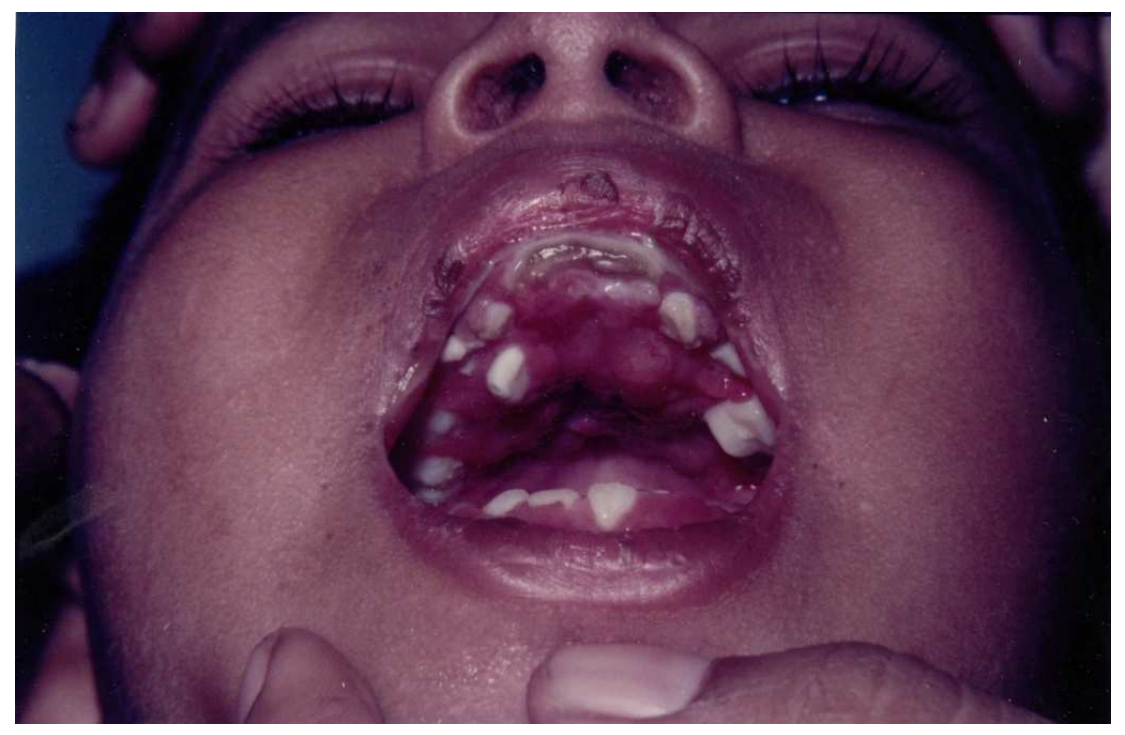

Fig1: Gingival growth with displaced teeth

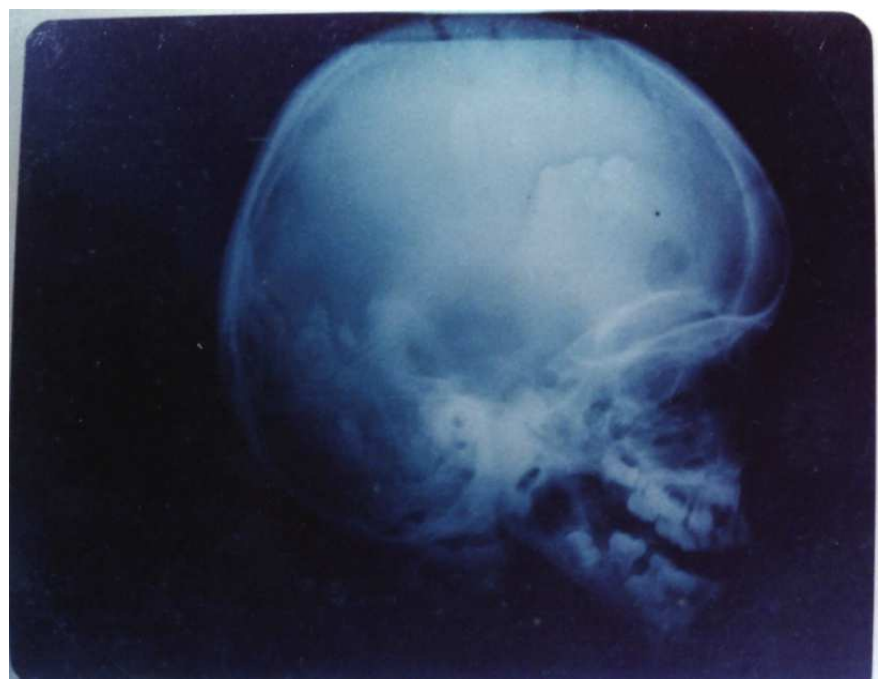

Fig 2: Radiolucent lytic lesion in skull 


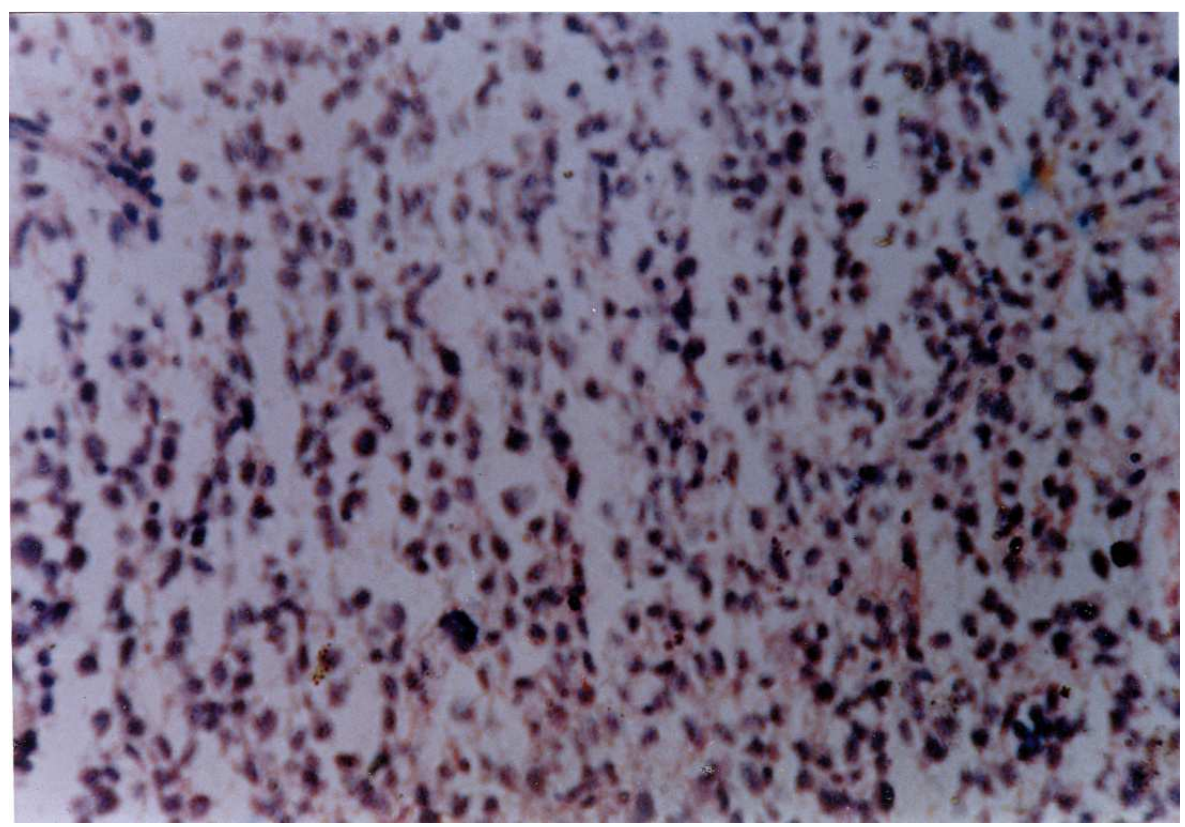

Fig 3: Histiocytes with pale cytoplasm and dark granules 